\title{
A Multi-Agent Approach to Segment Arabic Handwritten Text Lines
}

\author{
Mohsine Elkhayati, University Ibn Tofail, Morocco \\ Youssfi Elkettani, University Ibn Tofail, Morocco \\ Mohammed Mourchid, University Ibn Tofail, Morocco
}

\begin{abstract}
In text line segmentation, there are three classes of methods: either by sorting physical units such as pixels or connected components (CC) constituting a line or by searching for the baseline of each word and grouping together those who participate in the same line. The third class analyzes the separation locations between the lines. After an overview of lines segmentation approaches, the authors introduced a new method emphasizing its simplicity, speed, and originality. The proposed approach detects the starting components of the lines in the first step. In the second step, it defines a number of agents that start the segmentation process from their starting points between the starting components of lines. Each agent aims to reach the left edge of the document through the correct path. The algorithm used by the agents is based on the morphological process, characteristics of the Arabic manuscript and a communication system. The experimental results on an Arabic dataset show that this approach is an effective solution for the segmentation of lines from different Arabic manuscripts.
\end{abstract}

\section{KEYWORDS}

Connected Components, Diacritic Points, Handwritten Arabic Document, Intersection Points, Multi-Agent System, Overlapping Situation, Text Lines Segmentation

\section{INTRODUCTION}

In image processing, there are many recognition systems, among which there are Arabic handwriting recognition systems, which often consist of five steps: pre-processing, segmentation, feature extraction, recognition, and post-processing.

Segmentation is a procedure that divides or partitions an image into entities (words, subwords, paragraphs, characters, lines). This is an important phase because the segmentation rate has a direct influence on the rest of the character recognition process (Likforman-Sulem, Zahour, \& Taconet, 2007). In this work, the authors are interested only in the segmentation of handwritten Arabic documents into lines. Segmenting text lines consists of grouping all spatially aligned components (connected components, pixels, etc.).

The detection and segmentation of lines in printed documents is a less difficult task than that of handwritten documents. The extraction of lines from a handwritten text poses many challenges (Shi, Setlur, \& Govindaraju, 2009). Some of the challenges are: (1) variation of the inclination in the same line, (2) the large number of ascending and descending characters that produce overlaps between the adjacent lines, (3) variability of skew between text lines, (4) the presence of a large number of diacritic points in the inter-line spaces. 
The present work aims to segment the lines from handwritten Arabic documents by implementing an approach to deal with and solve the various problems mentioned (inclinations, overlaps, diacritics, etc.). In this context, the approach provides some techniques to deal with each situation in order to improve the segmentation rate and to minimize errors at the least cost.

Most of the existing approaches in the literature are based on algorithms providing specific processing for document analysis, i.e. using single processing for simple and complicated cases of the document. therefore, this results in a significant loss of time and memory space. After analyzing a large number of handwritten documents in Arabic, the authors found that most of the cases to be solved were simple, that they did not require many calculations and could be resolved quickly. Based on this principle, the authors have introduced a new approach based on a multi-agent system that can provide special and optimal treatment for each case: simple case, complicated and overlapping cases, which maximizes the speed of the algorithm, then save time and memory. More than its speed and simplicity, it should be mentioned that the approach is also robust because it can solve different situations and difficult problems.

The proposed approach is based on a multi-agent system, in which the main agent, also called Map agent (MA), creates a number of agents called snake agents (SA). Each SA starts its moving process from a starting position situated between two lines on the right edge of the document and attempts to reach the left edge of the document through the correct path. The SA agents can encounter three types of situations when moving: a simple situation, a complicated situation, and an overlapping situation. Each SA is equipped with an algorithm and a communication system that allows it to correctly manage each situation in order to make the right decision. When all SAs reach the edge of the document, the MA extracts the lines between the SA paths.

The rest of the paper is organized as follows: Section II examines some related works. Section III presents the challenges and motivations, Section IV describes the proposed approach. Section V presents the experimental results. Finally, Section VI concludes the paper.

\section{RELATED WORKS}

Generally, there are three classes of methods for segmenting text lines: either by sorting physical units such as pixels or connected components (CC) constituting a line or by analyzing the separation locations between the lines (Boulid, Souhar, \& Elkettani, 2016b). The third class looks for searching the baseline of each word and grouping together those that participate in the same line.

In another classification, Ouwayed and Belaïd (2012) grouped the methods of segmentation into three main classes: the ascending, descending and hybrid methods: the ascending methods are based on the grouping of basic components such as CC and the pixels in a line, which requires a lot of calculation and analysis. Descending methods rely on global characteristics to partition documents into homogeneous blocks. These methods are usually influenced by the overlap between lines. Hybrid methods combine the two previous classes to give better results. Razak, Zulkiflee, Idris, Noor, Salleh, and Yaacob (2008) presented a synthesis of existing line segmentation methods from the handwritten text.

Kumar et al. (2010) proposed an approach that consists of two main steps: Coarse text line estimation using primary components that define the line, and the assignment of diacritics to their associated line. After the deletion of the diacritic points, the algorithm starts with the estimation of the local orientation of each primary component, in order to build a similarity graph. The similarities between the components are calculated using the algorithm of the shortest path. From this stage, the lines are defined from two estimates obtained from Breadth-First Search, and affinity propagation. In the second step, diacritic points are reassigned to each line. The main advantage of the method is that lines of text with extremely small spacing can be segmented accurately, as well as lines of text with uniform and non-uniform asymmetry can be segmented correctly. On another side, the approach encounters problems in the extraction of diacritic points and the correction of touched components. 
The same authors (2011) proposed an approach that combines local and global techniques. Their work relies on a graph-based error detection technique and an Expectation-maximization (EM) -based correction technique. After filtering diacritical points and accents based on the mass, and size of the CCs, the direction of text-lines is estimated in each component. After that, a graph is constructed where the nodes correspond to components. In the next step, the Proximity errors and the components that touch or overlap are detected automatically based on the distances between the nodes in the local-orientation graph. At this point, the Expectation-maximization (EM) is Iteratively applied to split the touching lines. Finally, touching components are detected and split to obtain an accurate estimate of the line. The authors have tried to solve the problems of their previous method such as the detection of touching components and the extraction of diacritic points. This has improved the accuracy and speed of the method.

Boulid et al. (2016a) were based on the Markov decision process to detect Arabic handwritten text lines. The environment is represented by a set of components that are connected to each other. The algorithm starts with the detection of the component forming the beginning of the line and tries to find the next best component, until reaching the end of the line component. The algorithm is based on the field of vision as a displacement criterion and the Markov decision process (MDP) to make the decisions. The method is based on geometric information such as size, position, shape, and orientation of the $\mathrm{CC}$ which is better for complex documents, but it may be sensitive to changes in CCs' size and structure. The weakness of the method is that the problem of overlapping CCs has not been solved.

The same authors (2016b) proposed a new handwriting Arabic text line segmentation approach, based on a multi-agent system concept. The approach is inspired by the results observed in the human reading process. The idea is to reconstruct the lines of the text by detecting the components of each line in a successive manner. The system is composed of many agents, where each agent is designed to switch to a CC, to instantly see the other components of its field of vision, evaluate certain characteristics, determine and perform its behavior based on the results of its sensory feedback. Among the strong points of this approach, let us mention on the one hand the architecture based on a set of agents that allows preserving the traceability at each phase and allows going back to correct the errors and on the other hand, the presence of an agent responsible for the detection and segmentation of overlapping components.

Souhar et al. (2017) attempted to study the use of watershed transformation in the context of segmenting text lines from Arabic handwritten documents. They presented two approaches, the first is based on a local perspective, and the second is based on a global perspective. The segmentation process begins with detecting the locations of the text components in the document. Then it uses the watershed technique to partition the documents into well-defined areas. At the process's end, these areas are analyzed in order to classify them in well-defined lines. The approach with a global perspective achieved better results and showed its less sensitivity to the presence of noise and touching lines. In another side, the method needs some enhancements to prevent the broking of characters and to be able to treat historical and complex documents.

Inspired from human perception and common text characteristics, Daldali and Souhar (2018) proposed a Handwritten Arabic text line segmentation approach using seam carving. This technique is based on the notion of identifying high and low information areas on an image and takes the grayscale information as input instead of using a binary representation. The authors defined in the first stage the matched maximums between all of the document image slices, and then the piece-wise approximations are assembled by matching extremities into full line axis approximations. In the final stage, a set of operations are implemented in order to fuse discontinued lines and eliminate false positives. The use of seam carving with a global approach gives the method more flexibility to adapt to different languages and different situations. As inconvenient, the result of the method depends on the choice of the values of three parameters, which requires the development of an algorithm allowing to choose the values of these parameters according to the characteristics of each image. 
Al Dmour et al. (2018) proposed a new approach for extracting and straightening curvilinear text lines from handwritten Arabic documents. The authors first used a morphological dilation operation to extract text lines. In a second step, these lines were straightened using two operations: Course tuning of text lines orientation based on Hough transform, then fine tuning based on centroid alignment of the connected components that form the text line. The main advantage of the approach is that it can identify the skew angle, straightening the lines, and segmenting the multi-oriented and curvilinear text documents.

\section{CHALLENGES AND MOTIVATIONS}

\section{General Context of the Work}

Generally, optical character recognition systems (including Arabic) consists of five steps: preprocessing, segmentation, feature extraction, classification, and post-processing. This paper is part of a comprehensive work of Arabic handwriting recognition, which consists in the implementation of a framework allowing carrying out all the mentioned steps. After the implementation of a pre-treatment phase based on the method proposed by Boulid and Elkettani (2014), this paper addresses the text line segmentation phase by trying to bring a new approach that can improve the segmentation rate and minimize the time and space complexities.

\section{The Arabic Writing}

The Arabic language is one of the most used languages; it is the third most used language in the world. More than one billion Muslims use it as part of their religious activities. It is the main language of more than 250 million people, most of whom live in countries in the Middle East and North Africa. Several languages use Arabic alphabets, such as Urdu, Farsi (Persian), Pashtu, Jawi and Kurdish (Khorsheed, 2002).

Arabic script is written from right to left and is composed of 28 characters. It is cursive, that is to say, that the letters are generally related to each other. Each character can take four different forms, depending on its position in the word. A set of black pixels adjacent to each other is called a connected component. The latter, in the Arabic script, does not necessarily represent an entire word; it can be only a part of the word because certain characters should not be attached to their successor on the left in the word. On the other hand, there are different letters which have the same form, but which are distinguished by the position and the number of points which belong to them. The vowels "a", "i" and "o" are not used systematically in the Arabic script; signs that correspond to vowels are used to avoid pronunciation errors. Two types of texts can be distinguished: texts with or without vowel signs. Some Arabic texts (The Koran and the books of learning of reading and writing for children) contain vowel signs. The others, that is, books, newspapers, publications, are texts without these signs (Miled, Olivuer, Cherliet, \& Romeo-pakker, 1997).

\section{Challenges in Arabic Text Lines Segmentation}

Segmentation is an important phase in the process of recognizing Arabic handwriting. Segmenting a page includes two different phases: partitioning the page and segmenting words into characters: partitioning the page separates the different components of the page, such as text, figures, lines or paragraphs. The segmentation of the word is the extraction of the characters from the word (Shatnawi, 2015).

Since its cursive nature, the segmentation of lines from the Arabic manuscript text represents real challenges to the researchers (Li, Zheng, Doermann, \& Jaeger, 2008). Among the challenges, there are:

- Writing style that differs from one writer to another.

- The inclination in the lines. 
- The cursive style and calligraphy of Arabic writing.

- The presence of a large number of ascending and descending characters in the Arabic language.

- Overlaps between adjacent lines.

- The presence of diacritic points above and below the lines complicate the task.

Figure 1 shows some examples of these challenges.

Figure 1. Major problems of Arabic handwritten text-lines segmentation (a) tilt in the line (b) the presence of diacritical points between the lines (c) Overlaps between lines

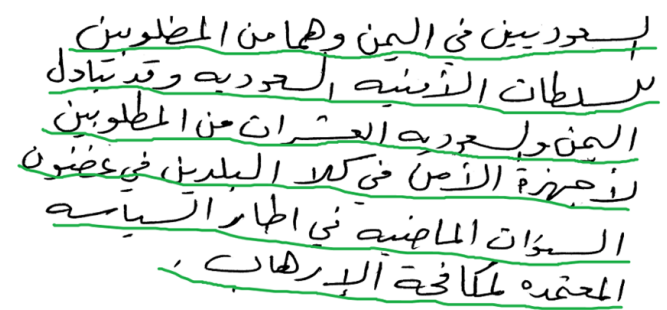

a

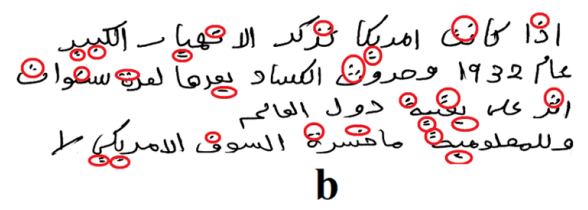

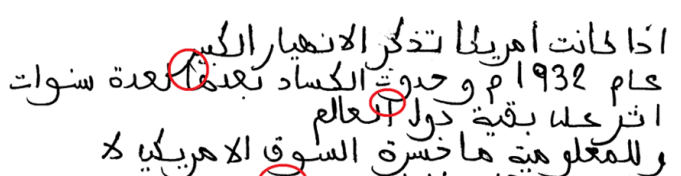
بئ

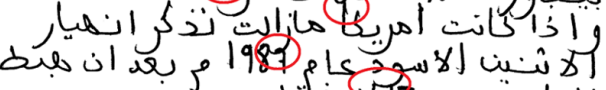

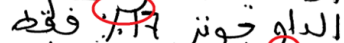

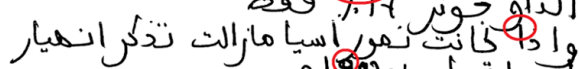

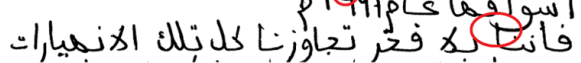

c

Several authors have attempted to solve a variety of these problems. In this context, several solutions are proposed in the literature, some are powerful and some are less effective. According to a literature review, it appears that the two major problems encountered by the researchers are the inclination of the lines and the overlap. In general, this document aims to propose a global approach that attempts to solve the various problems mentioned above. In order to achieve the best results for the segmentation of handwritten Arabic text lines. This approach provides special and robust techniques to overcome inclination problems, detect, and cut overlaps.

\section{PROPOSED APPROACH}

In the case of handwritten manuscripts in Arabic, writing is characterized by the notion of order and neighborhood, which means that the words and characters of a local region are more or less aligned on the same horizontal line, which is true even for curved writings (Boulid et al., 2016b). As mentioned in the previous section, Arabic is written from right to left and the text is represented in that order as a set of words or portions of words called Arabic word pieces (PAW). (Bouafif, Maddouri, \& Ellouze, 2012). The proposed approach is based on these principles and its implementation is described in this section.

In this approach, the authors use a multi-agent system. The main idea is to create a number of agents having a starting position and aiming to reach the edge of the document through the correct path. The algorithm used by the agents is based on morphological information, the characteristics of the Arabic handwritten text and a communication system between agents.

The principal agent MA is responsible for calculating the parameters that will be used in the segmentation algorithm. Based on these parameters, it removes the diacritical points and determines 
the starting components of the lines. According to this, it creates a number of SA agents, where each agent has a starting position between two adjacent lines, The SA searches for the correct path between two lines to reach the edge of the document using a specific algorithm. To ensure that all SAs are going from the right path, a communication system is set up between agents. For, for each difficulty, the SA can ask for help from the MA or its neighbours. All agents are reactive and goal oriented. Figure 2 illustrates the diagram of the interaction between the agents.

Bellow, an algorithm flow that attempts to summarize the main actions of the proposed system. The details and the processes of the actions will be explained in the following sections.

Algorithm

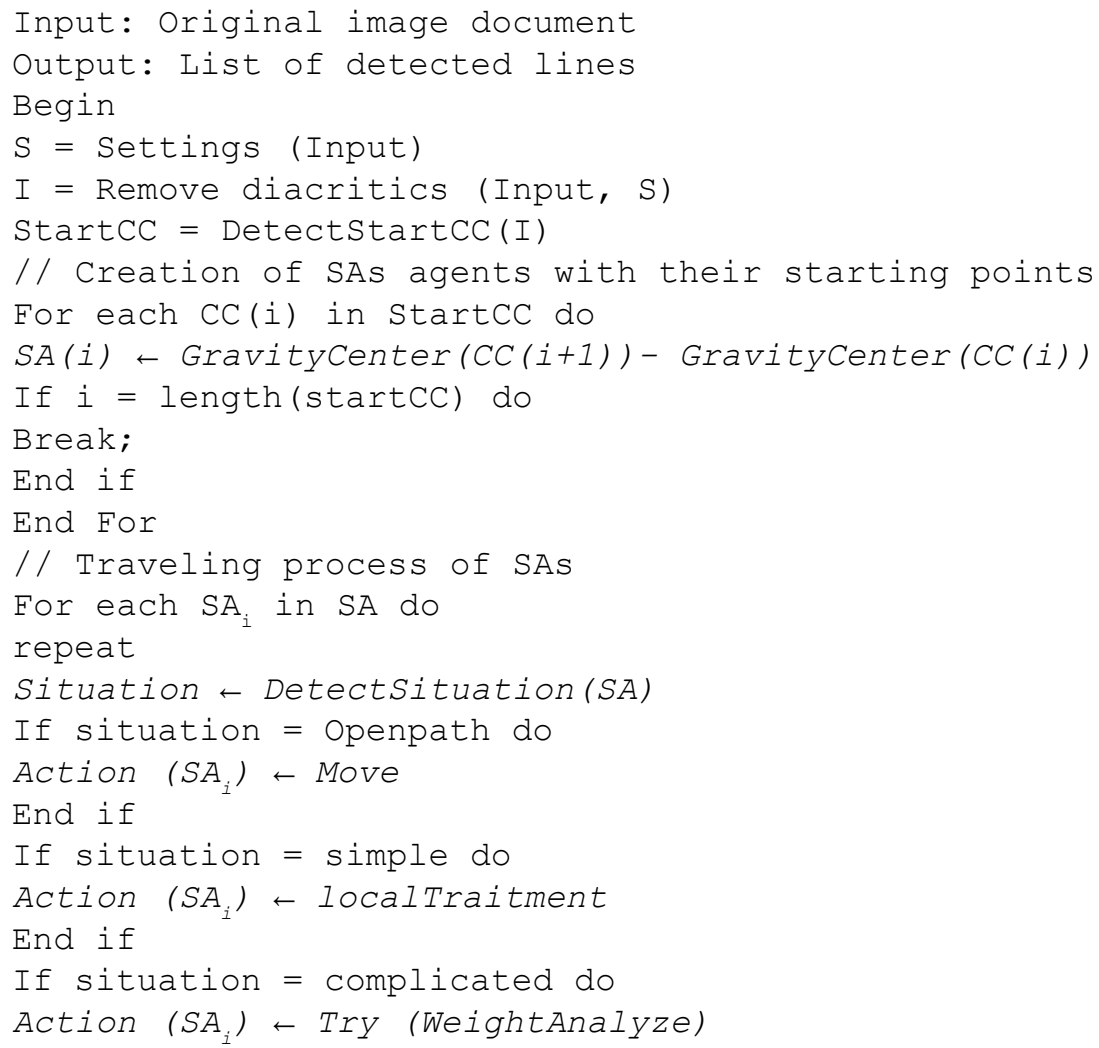

Figure 2. Block diagram illustrating interaction between agents

MAP Agent

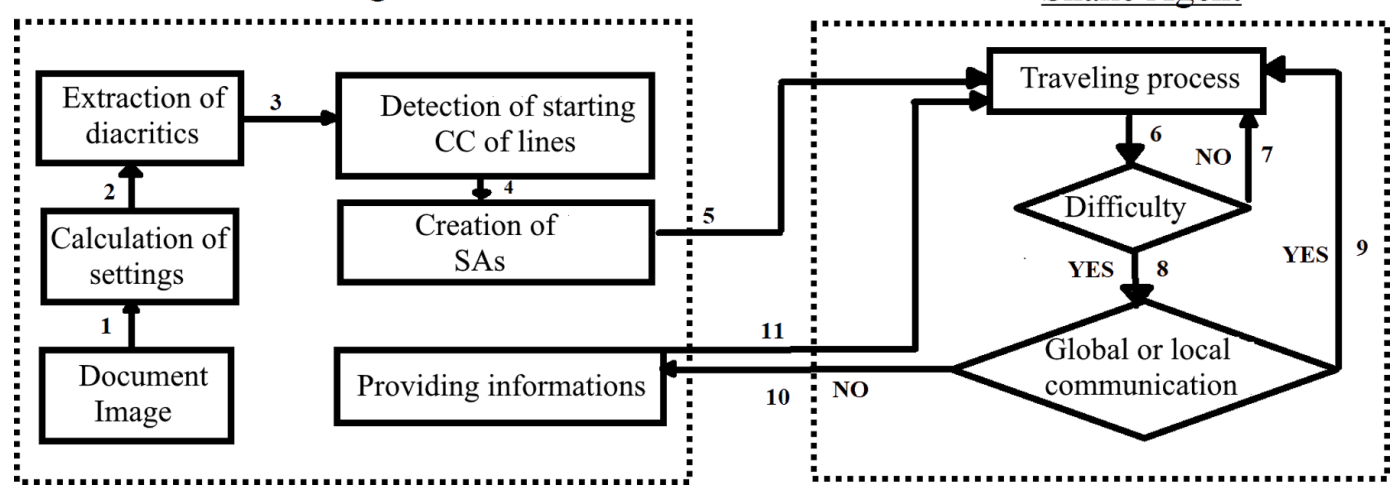




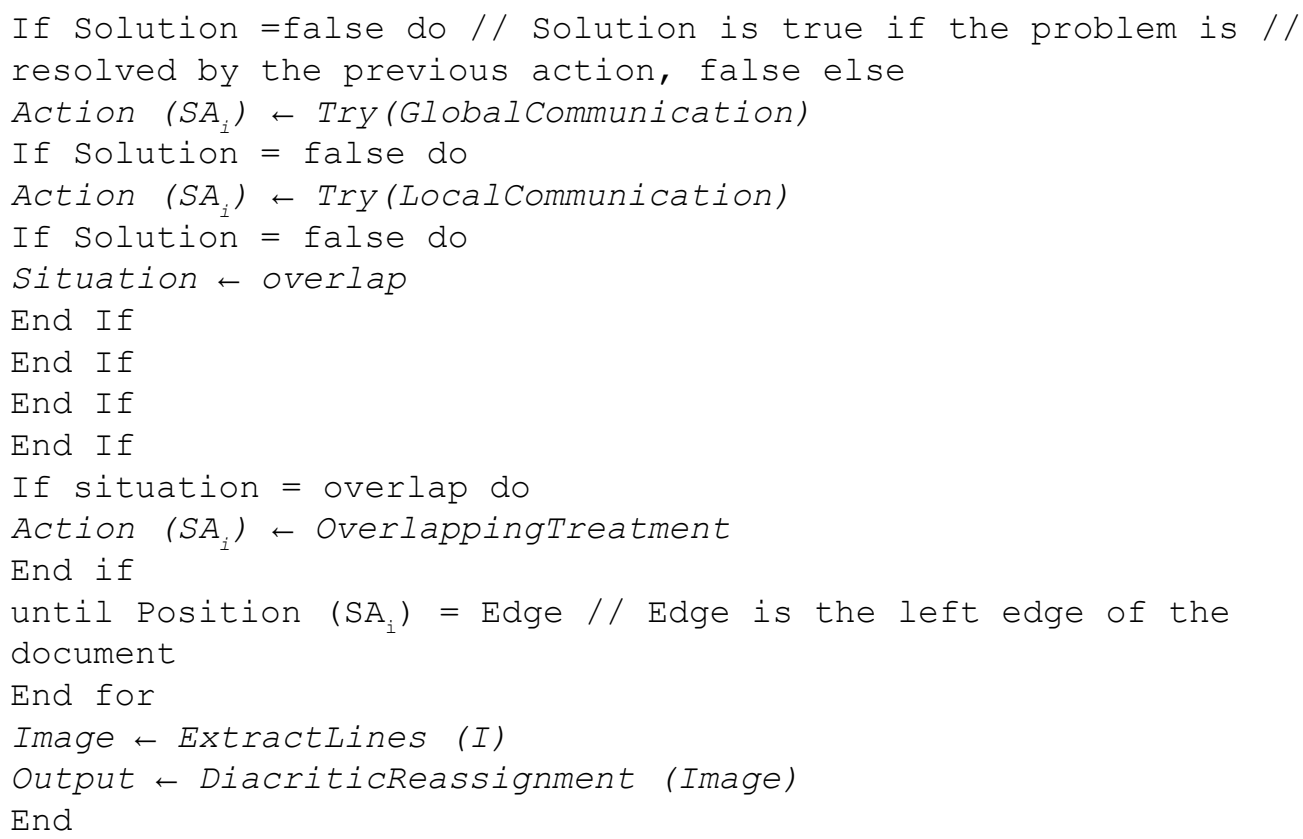

\section{Characteristics of the Environment}

For MA, the environment is fully observable because it must have total visibility of the image in order to calculate settings, remove diacritics, specify the first components of lines and resolve problems of all SAs. The SA is able to see only the path between two lines.

\section{Diacritics Suppression}

The presence of a large number of diacritical points can disrupt the process of extracting lines. To detect diacritical marks and dots, the MA calculates the global CCs parameters in the image to extract as many diacritical marks as possible without rejecting the text components. After several trials, the authors found that a CC can be considered as a diacritic point if its area is less than $40 *$ the average stroke width of the text. Diacritic marks and accents are saved for reassignment to the nearest text components at the process's end.

\section{Detection of First Components of Lines}

After extracting diacritics, the MA searches for the starting components of lines, it proceeds as follows: The agent loops through the image's CCs in rows and columns from top to bottom. If it finds a CC, it reframes a horizontal rectangle from the right edge of the $\mathrm{CC}$ to the right edge of the image. The $\mathrm{CC}$ is considered as the starting component of a line if $f(c c)=N P$ where:

$$
\begin{aligned}
& f(c c)=\sum_{x=T C}^{B C} \sum_{y=R C}^{R D} I(x, y) \\
& N P=\sum_{x=T C}^{B C} \sum_{y=R C}^{R D} 1
\end{aligned}
$$


With the notations, $T C$ : $y$-axis of top pixel of $C C ; B C$ : y-axis of bottom pixel of $C C ; R C$ : $\mathrm{x}$-axis of right pixel of $C C ; R D$ : x-axis of right pixel of the document; $N P$ : number of pixels in the rectangle and $I$ : the binary image.

The CCs with a solid rectangle in Figure 3 (a) represent the true starting CCs of lines.

After detecting the starting CCs of lines, the MA must create a set of agents (SA) between these CCs; every SA has a starting position defined as follows:

$$
S P(S A)=G C\left(c c_{\text {next }}\right)-\left(c c_{\text {current }}\right)
$$

With $S P(S A)$ is the starting position of SA, $G C(c c)$ is the y-axis of the gravity center of the CC. Figure 3 (b) shows examples of starting positions $(S P)$ of SA.

Figure 3. Illustration of the process of a) detecting the starting CCs and b) creating SAs with their starting positions

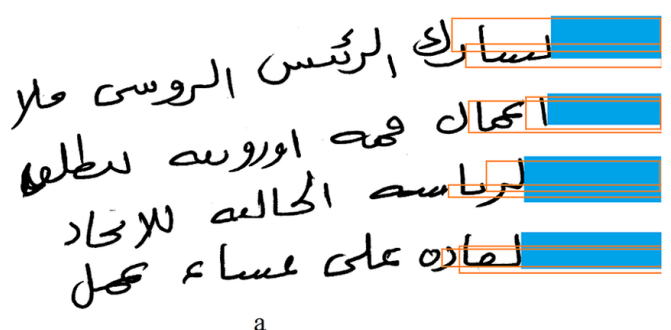

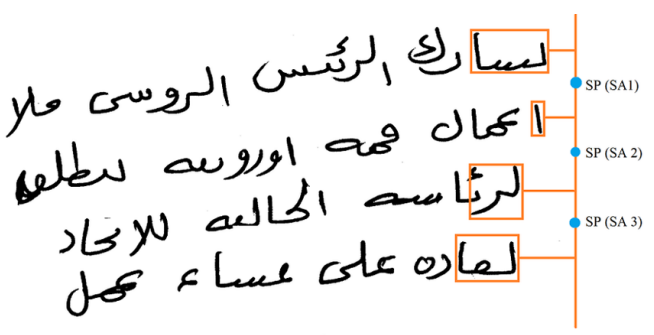

b

\section{SA's Moving Algorithm}

When an SA is created, it starts its moving process from its starting position. Its objective is to reach the edge of the document by passing through the correct path without cutting any $\mathrm{CC}$ and without crossing the path of another SA. To do this, all SAs are equipped with an algorithm to find the correct path and a communication system that allows exchanging information between neighbours and with the MA.

Using their starting positions, all SAs start their moving algorithms parallelly from right to left. Each SA continues its normal horizontal path to the image's left edge while it finds white pixels. But when it finds a black pixel, it must determine the nature of its situation (simple, complicated, overlapping) in order to provide the appropriate treatment. For this reason, a morphological analysis of the $\mathrm{CC}$ that obstructs the agent is required. The nature of the situation is determined according to the position of the SA in relation to the CC, as shown in Figure 4:

To know the nature of the situation, the SA calculates the height of the $\mathrm{CC}$, divides this height into thirds, and situate its position in relation to these thirds as shown in Figure 5.

Third $1=\bigcup_{i=T C}^{T C+h / 3} i$ 
Figure 4. Illustrations of possible situations according to SA's position relative to the component, (a) simple situation, (b) complicated situation, (c) overlapping situation

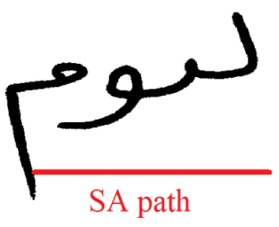

a

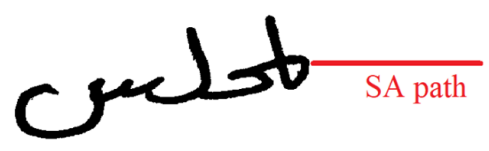

$\mathrm{b}$

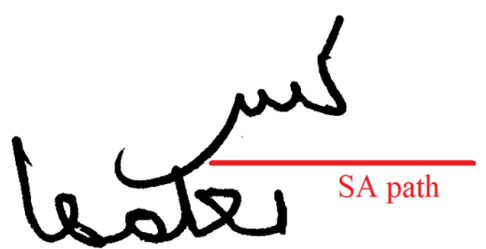

$\mathrm{c}$

Figure 5. Illustration of SA's position in relation to the CC
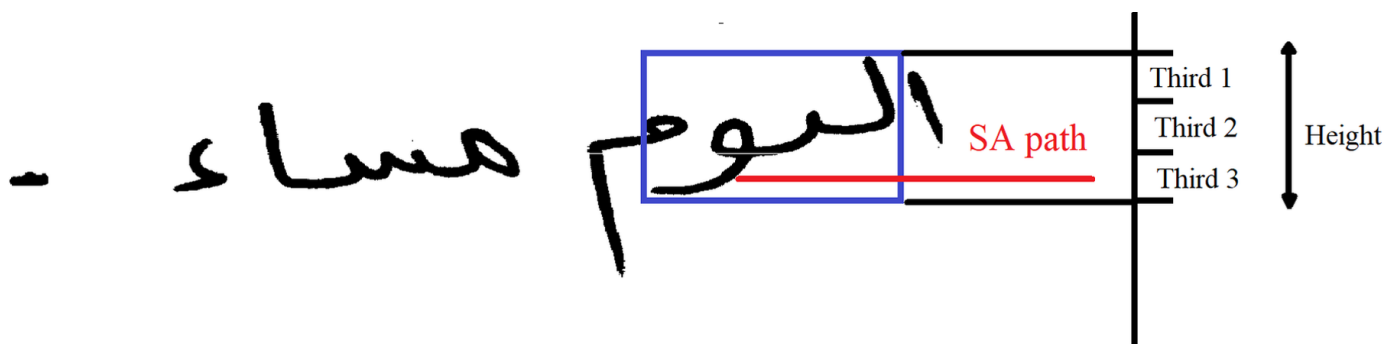

$\operatorname{Third} 2=\bigcup_{i=T C+h / 3}^{T C+2 h / 3} i$

$\operatorname{Third} 3=\bigcup_{i=T C+2 h / 3}^{T C+h} i$

Where $T C$ is the y-axis of the CC's top point and $h$ is the height of the CC.

If the horizontal position of SA belongs to Third 1 or Third 3, the SA considers that it is a simple situation; otherwise, if its position belongs to Third 2, it considers that it is a complicated situation. If the height of the current $\mathrm{CC}$ is greater than the average height of the text, the SA request information to know the paths of its neighbours. If its passage makes it cross the path of its two neighbours (successor and predecessor), it then declares an overlap situation (Figure 6). Depending on the nature of situation, the SA provides the appropriate treatment.

\section{Simple Situation}

As noted in the introduction, the authors found from the observation and analysis of a set of Arabic handwritten documents from several sources that most of the situations encountered in the documents are simple. Therefore, the goal of introducing three types of situations with appropriate treatments for each situation is to minimize computation time and memory allocation. Solving simple situations with simple processing achieves this goal.

In this case, the procedure is the simple one possible and requires only a very short time for calculation. If the position of SA belongs to Third 1, it passes over the CC; else, if its position belongs to Third 3, it passes below the CC. 


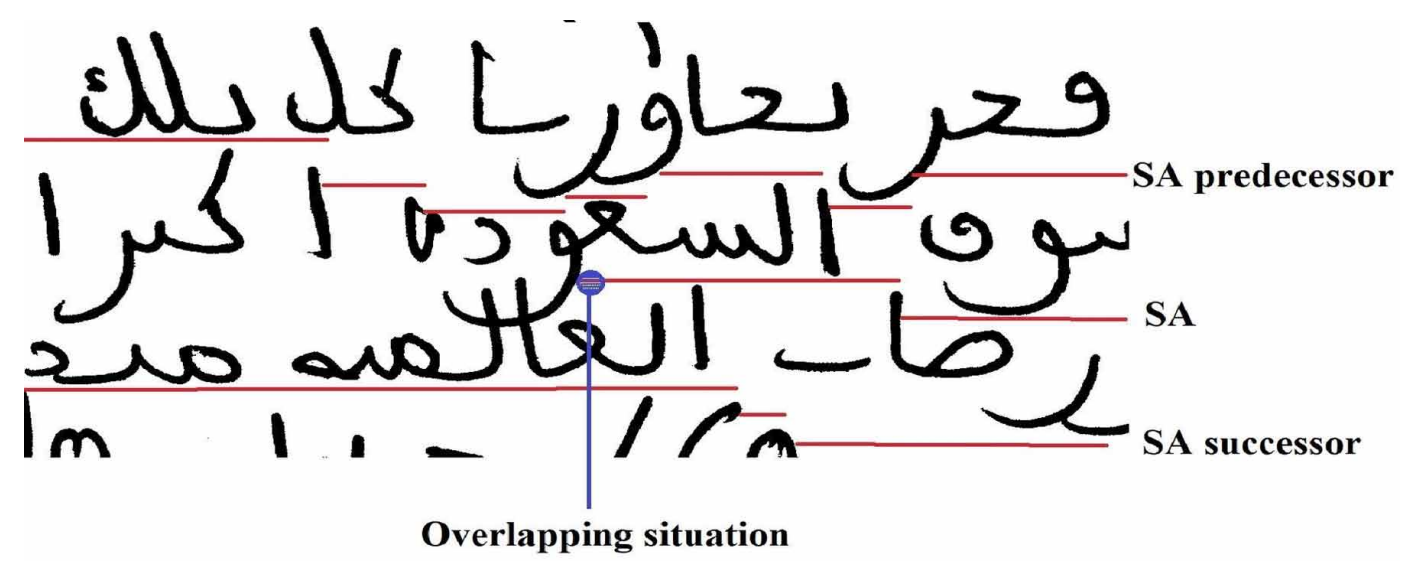

\section{Complicated Situation}

Even in complicated situations, the approach tries to minimize costs by gradually providing optimal treatments depending on the case. The principle is to try to make the decision using the simplest possible treatment. If a treatment fails to make an assured decision, the SA tries the next treatment.

In complicated situations, it is necessary to calculate other settings to make the right decision. First, the SA calculates and compares the weight of the top part and the bottom part of the CC. The weight of the rectangle is the number of black pixels it contains. Figure 7 shows an example of separation of a CC into a top and bottom part.

$$
\begin{aligned}
& W_{\text {top }}=\sum_{x=B C}^{P_{S A}} \sum_{y=L C}^{R C} I(x, y) \\
& W_{\text {bottom }}=\sum_{x=P_{S A}}^{B C} \sum_{y=L C}^{R C} I(x, y) \\
& W_{c c}=\sum_{x=T C}^{B C} \sum_{y=L C}^{R C} I(x, y)
\end{aligned}
$$

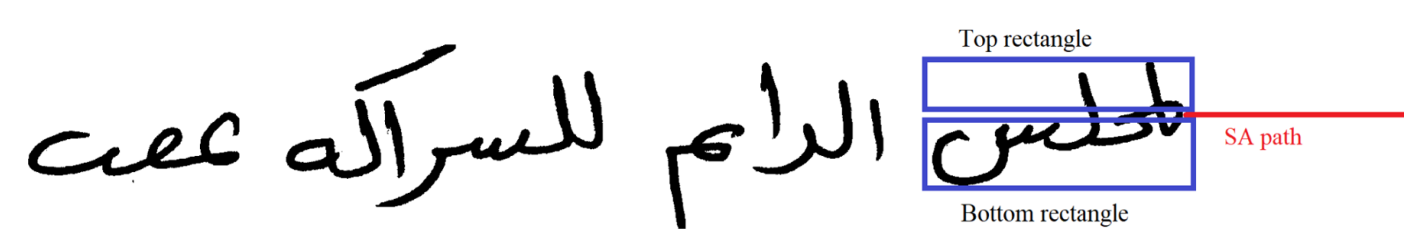


With $I(x, y)= \begin{cases}1 & \text { if the pixel isblack } \\ 0 & \text { else }\end{cases}$

With the notations, $W$ : the weight of CC; $P_{S A}$ : the y-axis of SA position; $T C$ : y-axis of CC's top point, $B C$ : $\mathrm{y}$-axis of CC's bottom point; $R C$ : $\mathrm{x}$-axis of CC's right edge; $L C$ : $\mathrm{x}$-axis of CC's left edge.

To make an assured decision, it is necessary that the weight of a part (top or bottom) must be greater than two-thirds of the CC's weight. For this reason, the following comparisons are done:

- If $W_{\text {top }}>2 * W_{c c} / 3$ that means the CC belongs to the top line, so the SA goes below the CC.

- If $W_{\text {bottom }}^{\text {top }}>2 * W_{c c} / 3$ that means the CC belongs to the bottom line, so the SA goes over the CC.

- Otherwise, SA establishes a communication with MA.

The MA constantly monitors all movements of SAs, it calculates the number of ascent and descent of all SAs. $N_{\text {ascent }}$ is the number of ascents of all SAs, $N_{\text {descent }}$ is the number of descents of all SAs.

When an SA needs for information it requests the MA to get $N_{\text {ascent }}$ and $N_{\text {descent }}$. If $N_{\text {ascent }}$ is dominant it means that the lines of text are upwards oriented, if the opposite, they are downwards oriented. Figure 8 shows two types of text (a) upward oriented, and (b) downward oriented.

In the same way as the previous treatment, to have an assured decision, it is necessary that one of the variations ( $N_{\text {ascent }}$ or $N_{\text {descent }}$ ) must be greater than two-thirds of the total number of variations. To check that, the following treatment is done:

Figure 8. Example of SA's ascents and descents; (a) upward oriented text; (b) downward oriented text.

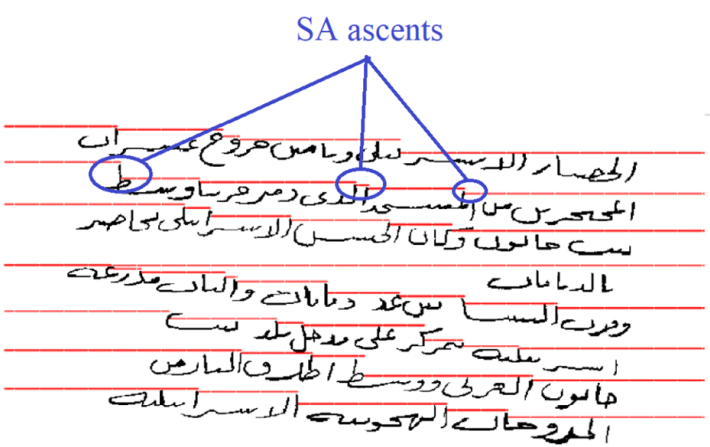

a

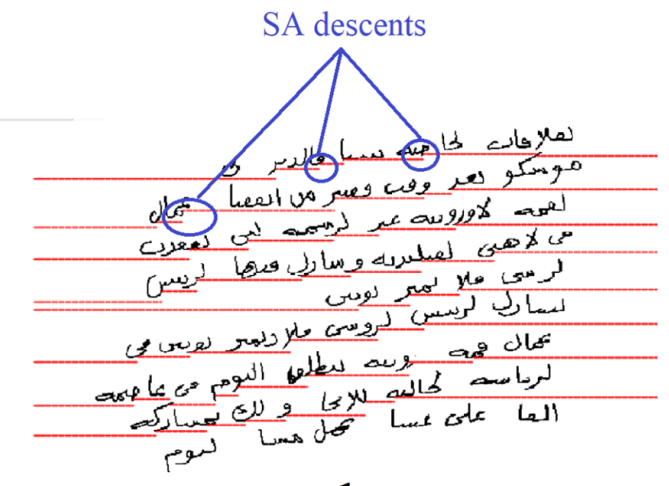

b

$N_{\text {variation }}=N_{\text {ascent }}+N_{\text {descent }}$

- If $N_{\text {ascent }}>2 * N_{\text {variation }} / 3$ that means the CC belongs to the bottom line, so the SA goes over the CC.

- If $N_{\text {descent }}>2 * N_{\text {variation }} / 3$ that means the CC belongs to the top line, so the SA goes below the CC.

Finally, if it is not resolved, the SA establishes a local communication with its neighbors in order to know the vertical parallel positions of its successor and its predecessor. Figure 9 shows a situation that needs communication between SAs. 
Based on the position of its neighbours, the SA decides whether to go up or down. If its ascent or descent makes it cross the path of its predecessor or successor, it cancels the decision. In the case where it finds no empty path, it declares an overlapping situation.

Figure 9. Example of a situation that needs communication between neighbours

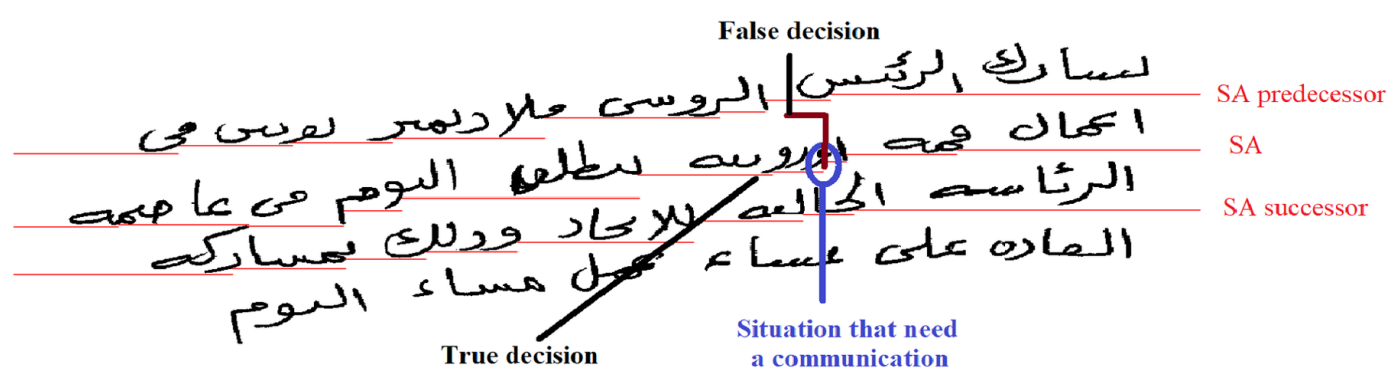

\section{Overlapping Situation}

The presence of overlapping or touching components is a real challenge for text line segmentation algorithms. These components can disrupt the segmentation process and lead to serious errors in the subsequent recognition process. For this reason, the proposed approach has attempted to detect this type of components in order to cut them.

According to the nature of the Arabic script which is characterized by the presence of the ascenders and descenders, overlaps between characters or words often occur in interline spaces. Therefore, in first stage, SA applies a horizontal projection profile on a part of the line (two adjacent left and two right components) to detect the area of interest. In the second stage, it tries to find an open path in this area and continues its moving process until reaching a closed path (overlapping area). Figure 10 (a) shows the process to reach an overlapping area.

At this point, a vertical projection is applied on a part of $\mathrm{CC}$ from the SA position until finding two local minima (one on the right and one on the left). Next, the rectangle constructed from the intersection of the areas detected by the horizontal and vertical projections will be extracted, as shown in Figure 10 (b).

In order to locate the positions of gaps, the idea is to analyze the continuity of the stroke in the region of interest and to disconnect the non-linear parts. For this reason, and after detecting the area

Figure 10. Process to detect and extract the overlapping zone

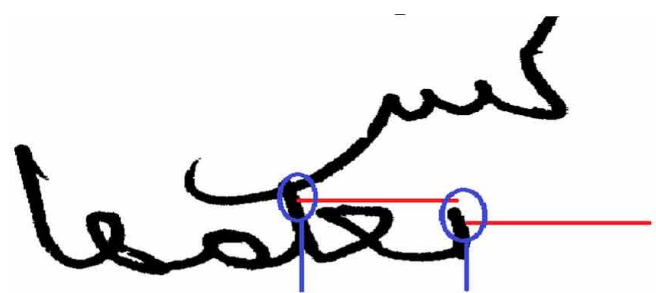

Closed path Open path

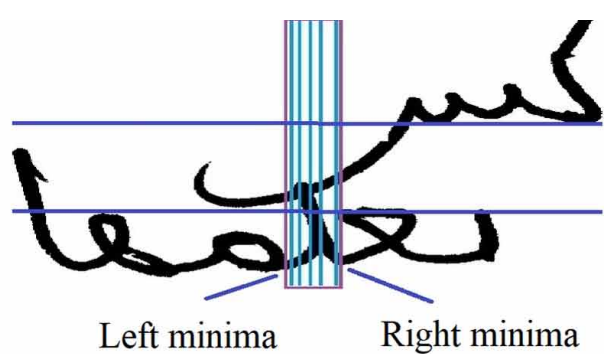

b 
of interest, the approach provides an algorithm for segmenting overlapping components, based on the following steps:

Step 1: Extract the skeleton from the rectangle.

Step 2: Detect the intersection points in the skeleton that correspond to pixels connected to three or more pixels.

Step 3: Erode the position of these points on the original component with a structuring element disk equal to the average line width, which allows SA to find an open path.

Step 4: Passage of SA through the open path.

Step 5: Once SA has made its passage, the eroded pixels above and below the SA's path will be filled.

Like the algorithms for simple and complicated situations, the algorithm for detecting and cutting overlapping components is also applied by SA. Figure 11 illustrates the process of cutting an overlapping CC.

\section{Extraction of Lines}

When all the SAs reach the edge of the document, the MA agent extracts the CCs between the path of the first SA and the path of the next SA. This treatment is executed in an iterative manner for each line until there are no more lines to be extracted. The diacritics are reassigned to its closest component.

Figure 11. Process of cutting an overlapping $\mathrm{CC}(\mathrm{a})$ extraction of the skeleton; (b) detection of the intersection points; (c) erosion of the position of intersection points in the original image; (d) passage of SA; (e) filling the pixels above and below the SA path.

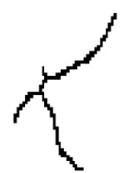

a

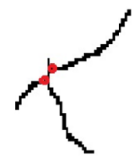

$\mathrm{b}$

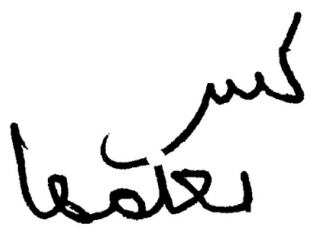

$\mathrm{c}$

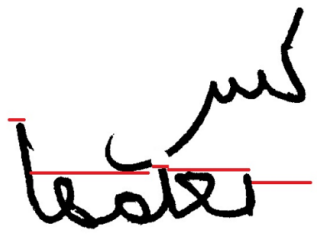

d

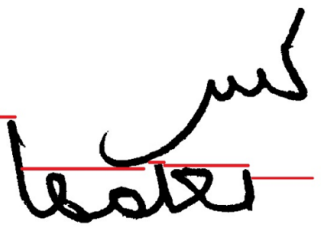

$\mathrm{e}$

\section{EXPERIMENTATION AND EVALUATION}

\section{Database}

To evaluate the performance of the approach, two handwritten Arabic databases are used: KFUPM Handwritten Arabic Text (KHATT) (Mahmoud et al. 2014), and Handwritten Arabic Proximity Datasets (HAPD) (Language and Media Processing Laboratory, n.d.).

KHATT is an open access database that provides researchers with basic data for testing and improving the quality of their techniques and tools in research areas dealing with handwritten documents in Arabic. The database contains 2000 paragraphs of minimum text covering all forms of Arabic characters and 2000 paragraphs randomly selected from 46 sources. The 2000 paragraphs of random text consist of 9327 lines.

The Arabic handwritten proximity dataset is specially trained for detecting lines of text. It contains a set of images with spaces of different sizes between the text lines. These images show different levels of overlap and touching between text lines, from null to severe. They are generated from a set of base images by moving the lines of text close to those above. 
The experimentation is performed on 2000 images containing 9327 lines from KHATT and 125 images containing 1974 lines from HAPD.

\section{Programming Platform}

The algorithm was implemented using MATLAB (R 2017a). All experiments are performed on a computer with an i-3-3110M processor with a frequency of $2.40 \mathrm{GHz}, 4 \mathrm{~GB}$ RAM, and Windows 10 64-bit operating system.

\section{Evaluation Measures}

To evaluate the performances of the proposed method, the authors used the following criteria: F1score to specify the segmentation rate, time to specify the time complexity of the approach, allocated and freed memory to specify the space complexity of the approach.

The F1-score is a technique that evaluates approaches. This evaluation technique is based on the calculation of the number of correspondences between the lines extracted by the system and the existing lines in the ground truth. Matching score (MS) is the criterion used for this reason, its values are calculated from the intersection of the pixels obtained on the result of the segmentation process with those of the ground truth (Phillips, \& Chhabra, 1999). MS is calculated as follows:

$$
\operatorname{MS}\left(r_{i}, g_{j}\right)=\frac{T\left(P\left(r_{i}\right) \bigcap P\left(g_{j}\right)\right)}{T\left(P\left(r_{i}\right) \bigcup P\left(g_{j}\right)\right)}
$$

With $M S(r i, g j)$ is a real number between 0 and 1 which represents the matching score between the zone $r_{i}$ resulted from the algorithm and the zone $\mathrm{g}_{\mathrm{j}}$ in the ground truth. $P$ correspond to pixels that represent the foreground (text) and $T$ is an operator that counts the number of pixels in each zone.

To obtain the score between all the zones obtained by the method, and the existing zones on the ground truth, the following treatment is done: If the resulted zones do not match any zone in the ground truth it is counted as false positive $(F P)$. If the score for a zone is found above a threshold, it is counted as true positive $(T P)$. The zones in the dataset that are left unmatched are considered false negative $(F N)$. Therefore, precision, recall, and F1-score are counted as follows:

$$
\text { precesion }=\frac{T P}{T P+F P}
$$

$$
\text { recall }=\frac{T P}{T P+F N}
$$

$$
F 1_{\text {score }}=\frac{2 * \text { precision } * \text { recall }}{\text { precision }+ \text { recall }}
$$




\section{RESULTS, COMPARISON AND DISCUSSION}

\section{Space and Time Complexity}

The performance of a segmentation algorithm is not only measured by the extraction rate or the error rate, but also by other measures such as time and space complexities. This section shows and discusses the performance of the proposed algorithm in the light of these two metrics.

It should be mentioned that the time and space complexities of an algorithm are not stable values, but vary according to several criteria such as the performance of the test machine, the size of the image to be processed, the number of overlaps in the text, the inclination of the text, the number of $\mathrm{CC}$ in the text. etc. However, the test is performed on 20 images with size $1000 * 1224$ and average criteria (overlaps, inclinations, number of $\mathrm{CC}$ ) using the machine with the characteristics mentioned in the previous section. Table 1 shows the average time used, the average allocated memory, and the average freed memory by the algorithm and by each of its stages.

From Table 1, it appears that the main phase of the algorithm "Moving process" containing all the appropriate treatments for each situation takes only a similar or less time compared to the time required for the preparatory phases such as the "calculation of parameters "and" the extraction of diacritics “.

Table 1. Spatial and temporal complexity of the proposed algorithm and the techniques it contains

\begin{tabular}{|l|l|l|l|l|}
\hline Algorithm \& stages & Type of stage & Time & Allocated memory & Freed memory \\
\hline $\begin{array}{l}\text { The Complete } \\
\text { algorithm }\end{array}$ & complete & $3.344 \mathrm{~s}$ & $455064.00 \mathrm{~Kb}$ & $446632.00 \mathrm{~Kb}$ \\
\hline $\begin{array}{l}\text { Calculation of } \\
\text { settings }\end{array}$ & preparatory & $1.304 \mathrm{~s}$ & $14796.00 \mathrm{~Kb}$ & $6364.00 \mathrm{~Kb}$ \\
\hline $\begin{array}{l}\text { Extraction of } \\
\text { diacritics }\end{array}$ & preparatory & $1.151 \mathrm{~s}$ & $39600.00 \mathrm{~Kb}$ & $17216.00 \mathrm{~Kb}$ \\
\hline $\begin{array}{l}\text { Detecting starting CC } \\
\text { of lines }\end{array}$ & preparatory & $0.381 \mathrm{~s}$ & $15496.00 \mathrm{~Kb}$ & $15496.00 \mathrm{~Kb}$ \\
\hline $\begin{array}{l}\text { Moving process of } \\
\text { all SAs }\end{array}$ & main & $1.167 \mathrm{~s}$ & $376596.00 \mathrm{~Kb}$ & $376596.00 \mathrm{~Kb}$ \\
\hline $\begin{array}{l}\text { Note: } \\
\text { - Time: is the cumulative time used by an algorithm during all its executions. Note that it is possible for an algorithm to run multiple times if necessary. } \\
\text { - Allocated memory: is the total cumulative amount of memory allocated during the execution of an algorithm. } \\
\text { - Freed memory: is the total cumulative amount of memory de-allocated during the execution of an algorithm. }\end{array}$
\end{tabular}

About the space complexity, it appears that the algorithm allocates a reasonable space with respect to the amount of information processed. If we differentiate between the allocated memory and the freed memory by the algorithm (11 MB), we can deduce that the execution of the algorithm does not require a lot of memory and that it can be executed even with inefficient machines. We also observe that the allocated memory and the freed memory by the main phase of the algorithm "displacement process" are equal, which reinforces the idea that the preparatory phases are responsible for the consumption of the memory space.

The obtained results show that an approach offering specific treatments depending on the situation, from the simplest to the most complicated, could be an effective solution to the problems of time and space. Moreover, it is possible to say that the preparatory phases of the algorithm consume more time and space than the main phase, which encourages the development of strategies to improve these 
phases at this level. Finally, and from this experiment we can deduce that the algorithm showed its spatio-temporal performances.

\section{Segmentation Rate}

To test the performance of the proposed method, the $90 \%$ and $95 \%$ values are used for the MS-threshold criterion. The reason for this choice is that most of the work in this direction has used these two values, which makes it possible to compare the results obtained with other results of the literature. Table 1 shows the results obtained by testing the method on both databases.

Table 2. Results obtained from the test of the approach on KHATT and HAPD databases

\begin{tabular}{|l|l|l|}
\hline \multirow{2}{*}{ Database } & F1-score & MS=95 \\
\cline { 2 - 3 } & MS=90 & $97.5 \%$ \\
\hline KHATT & $99.1 \%$ & $98.9 \%$ \\
\hline HAPD & $99.4 \%$ & \\
\hline
\end{tabular}

Table 1 shows that the results obtained by the method are satisfactory on both databases especially for the MS $=90$ the F1-score exceeds $99 \%$.

To compare the performance of the proposed method with other methods in the literature, the authors used the results obtained from the HAPD dataset, which is frequently used by researchers in the literature. Table 2 compares the score of the proposed approach with other approaches tested on the same dataset.

The comparison presented in Table 3 shows that the proposed method has given satisfactory results compared to other methods from the literature. These results prove the effectiveness of the method and its ability to adapt to different styles of Arabic writing and its ability to solve different problematic situations. The results also show that the approaches that include techniques for solving overlap problems (methods $2,5,7,8$ ) have performed better than those that have not (methods 1 , 3,6 ). This may allow saying that the proposed method provides an efficient technique for detection and segmentation of overlaps.

It can also be said that the method made it possible to minimize segmentation errors $(0.6 \%$ for MS $=90$ and $1.1 \%$ for MS =95). The presence of a large number of ascenders and descenders in

Table 3. Comparison of Results obtained on HAPD dataset

\begin{tabular}{|l|l|l|l|l|}
\hline \multirow{2}{*}{$\mathbf{N}^{\circ}$} & \multirow{2}{*}{ Authors } & Methods & \multicolumn{2}{|l|}{ F1-score } \\
\cline { 4 - 5 } & & & MS=90 & MS=95 \\
\hline 1 & (Kumar et al., 2010) & Affinity propagation & $95.6 \%$ & $90.9 \%$ \\
\hline 2 & (Kumar et al., 2011) & Expectation-Maximization & $98.8 \%$ & Not reported \\
\hline 3 & (Zhang, \& Tan, 2014). & constrained seam carving & $98.41 \%$ & Not reported \\
\hline 4 & (Boulid et al., 2016a) & Field of vision (Marcov decision process) & $95.8 \%$ & $90.5 \%$ \\
\hline 5 & (Boulid et al., 2016b) & Field of vision (Multi-Agent system) & $97.4 \%$ & $94.3 \%$ \\
\hline 6 & (Souhar et al., 2017) & Watershed Transform & Not reported & $93.3 \%$ \\
\hline 7 & (Daldali, \& Souhar, 2018) & Seam carving & $97.5 \%$ & $93.4 \%$ \\
\hline 8 & Elkhayati et al. & The proposed method & $99.4 \%$ & $98.9 \%$ \\
\hline
\end{tabular}


handwritten documents, the complex overlap of lines, and the large inclinations in lines are generally the factors responsible for erroneous decisions made by agents, which produce segmentation errors. Through a visual observation of the results, it appears that most of the errors produced are usually small ascending or descending CCs that are attached to a false line. On the contrary, false segmentations of complete lines are rare.

The method provides adapted techniques based on the characteristics of the Arabic script which allowed it to obtain excellent results on two different databases. The authors imagine that the generalization of the approach to other manuscripts may yield good results, but not to the same extent because the approach holds its strength from the focus on the details of the Arabic manuscript. In this sense, future work will aim to generalize the approach so that it builds on the common characteristics of a set of manuscripts from the same family in order to see the results that can be obtained.

Figure 12 illustrates some visual results of the approach. Figure 12. Illustration of some visual results of the approach

\section{CONCLUSION}

In this paper, the authors presented a new approach for the segmentation of lines from handwritten texts in Arabic. The approach is based on a multi-agent system in which all agents collaborate to achieve their ultimate goal: "reach the edge of the document through the correct path". All agents use an algorithm based on a progressive process that allows making the best decision at the lowest cost. The strength of the algorithm comes from the fact that it can provide different treatments depending on each situation, which minimizes the calculation time and save memory space. The treatments provided by the algorithm are based on the analysis of inter-line spaces, the morphological conditions and the characteristics of the Arabic script.

The method was tested on two databases containing Arabic manuscript documents from several sources, written by different authors and styles. Both databases contain a large number of overlapping and touching lines, they also contain lines showing different degrees of inclination and orientation. From the obtained results and the comparison with other methods of the literature It can be said that the method has proved its effectiveness, its ability to adapt to different styles of Arabic writing and its ability to solve a large number of problems. Moreover, the experimentation on space and time complexities of the approach has shown its speed and its capacity to solve each situation (simple, complicated, overlapping) with the lowest possible cost.

In future works, the authors intend to improve this approach on a few points, adding more robustness by implementing a correction cycle to detect false segmentions and correct them, a more intelligent approach to cut an overlapping component, and finally generalize the approach on other manuscripts. 


\section{REFERENCES}

Bouafif, F., Maddouri, S., \& Ellouze, N. (2012). A hybrid method for three-segmentation level of handwritten Arabic script. The International Arab Journal of Information Technology, 9(2), 117-123.

Boulid, Y., \& Elkettani, M. Y. (2014). Approach for Arabic Handwritten Image Processing: Case of Text Detection in Degraded Documents. International Journal of Computers and Applications, 101(14).

Boulid, Y., Souhar, A., \& El Kettani, M. E. Y. (2016a). Detection of Text Lines of Handwritten Arabic Manuscripts using Markov Decision Processes. International Journal of Interactive Multimedia and Artificial Intelligence, 4(1), 31-36. doi:10.9781/ijimai.2016.416

Boulid, Y., Souhar, A., \& Elkettani, M. Y. (2016b). Segmentation approach of Arabic manuscripts text lines based on multi agent systems. International Journal of Computer Information Systems and Industrial Management Applications, 8, 173-183.

Daldali, M., \& Souhar, A. (2018). Handwritten Arabic documents segmentation into text lines using seam carving. International Journal of Interactive Multimedia and Artificial Intelligence, 5(5), 89-96. doi:10.9781/ ijimai.2018.06.002

Khorsheed, M. (2002). Off-Line Arabic Character Recognition - A Review. Pattern Analysis \& Applications, 5(1), 31-45. doi:10.1007/s100440200004

Kumar, J., Abd-Almageed, W., Kang, L., \& Doermann, D. (2010, June). Handwritten Arabic text line segmentation using affinity propagation. In Proceedings of the 9th IAPR International Workshop on Document Analysis Systems (pp. 135-142). ACM.

Kumar, J., Kang, L., Doermann, D., \& Abd-Almageed, W. (2011, September). Segmentation of handwritten textlines in presence of touching components. In Proceeding of the International Conference on Document Analysis and Recognition (ICDAR) (pp. 109-113). IEEE. 10.1109/ICDAR.2011.31

Language and Media Processing Laboratory. (n.d.). Handwritten Arabic Proximity Datasets. Retrieved from https://lampsrv02.umiacs.umd.edu/projdb/project.php?id=65

Li, Y., Zheng, Y., Doermann, D., \& Jaeger, S. (2008). Script-independent text line segmentation in freestyle handwritten documents. Pattern Analysis and Machine Intelligence. IEEE Transactions on, 30(8), 1313-1329.

Likforman-Sulem, L., Zahour, A., \& Taconet, B. (2007). Text line segmentation of historical documents: A survey. International Journal on Document Analysis and Recognition, 9(2-4), 123-138. doi:10.1007/s10032-006-0023-Z

Mahmoud, S., Ahmad, I., Al-Khatib, W., Alshayeb, M., Tanvir Parvez, M., Märgner, V., \& Fink, G. A. (2014). KHATT: An Open Arabic Offline Handwritten Text Database. Pattern Recognition, 47(3), 1096-1112. doi:10.1016/j.patcog.2013.08.009

Miled, H., Olivier, C., Cheriet, M., \& Romeo-Pakker, K. (1997). Une Méthode Rapide de Reconnaissance de l'Écriture Arabe Manuscrite. In Proceeding of Seizième colloque GRETSI (pp. 857-860). Academic Press.

Ouwayed, N., \& Belaïd, A. (2012). A general approach for multi-oriented text line extraction of handwritten documents. International Journal on Document Analysis and Recognition, 15(4), 297-314. doi:10.1007/s10032011-0172-6

Phillips, I. T., \& Chhabra, A. K. (1999). Empirical performance evaluation of graphics recognition systems. IEEE Transactions on Pattern Analysis and Machine Intelligence, 21(9), 849-870.

Razak, Z., Zulkiflee, K., Idris, M. Y. I., Tamil, E. M., Noor, M. N. M., Salleh, R., \& Yaacob, M. (2008). Off-line handwriting text line segmentation: A review. International journal of computer science and network security, $8(7), 12-20$.

Shatnawi, M. (2015). Off-line handwritten Arabic character recognition: a survey. In Proceedings of the international conference on image processing, computer vision, and pattern recognition (p. 52). IPCV.

Shi, Z., Setlur, S., \& Govindaraju, V. (2009). A Steerable Directional Local Profile Technique for Extraction of Handwritten Arabic Text Lines. In Proceeding of International Conference on Document Analysis and Recognition (pp. 176-180). Academic Press; . doi:10.1109/ICDAR.2009.79 
Souhar, A., Boulid, Y., Ameur, E., \& Ouagague, M. (2017). Segmentation of Arabic Handwritten Documents into Text Lines using Watershed Transform. International Journal of Interactive Multimedia and Artificial Intelligence, 4(6), 96-102. doi:10.9781/ijimai.2017.08.002

Zhang, X., \& Tan, C. L. (2014, September). Text line segmentation for handwritten documents using constrained seam carving. In Proceeding in the 14th International Conference on Frontiers in Handwriting Recognition (ICFHR) (pp. 98-103). IEEE. 10.1109/ICFHR.2014.24 